\title{
Coulombic couplings between pigments in the major light-harvesting complex LHC II calculated by the transition density cube method
}

\author{
Jan S. Frähmcke ${ }^{a}$, Peter J. Walla ${ }^{a, b, *}$ \\ ${ }^{a}$ Technical University of Brunswick, Institute for Physical and Theoretical Chemistry, Department for Biophysical Chemistry, \\ Hans-Sommerstr. 10, D-38106 Braunschweig, Germany \\ ${ }^{\mathrm{b}}$ Max-Planck-Institute for Biophysical Chemistry, Department of Spectroscopy and Photochemical Kinetics Am Faßberg 11, D-37077 Göttingen, Germany
}

Received 4 August 2006; in final form 31 August 2006

Available online 9 September 2006

\begin{abstract}
We present ab initio transition density cube (TDC) calculations of the coulombic couplings between chlorophyll and carotenoid pigments in the major light-harvesting complex of photosystem II (LHC II) based on the $2.72 \AA$ structure [Liu et al., Nature 428(2004) 287292]. A comparison with couplings calculated by the ideal dipole approximation (IDA) demonstrate that for inter-pigment distances of less than $\sim 25 \AA$ the IDA-values can deviate by up to one order of magnitude from the exact values calculated by the TDC-method. The largest deviations are observed for interactions involving $\mathrm{Q}_{x}$ states because of a significant multipole character of the corresponding $\mathrm{Q}_{x}$ transitions.
\end{abstract}

(C) 2006 Elsevier B.V. All rights reserved.

\section{Introduction}

The most abundant pigment-protein complex in the photosynthetic apparatus of plants and green algae is the major light-harvesting protein LHC II of photosystem II. This pigment-protein complex probably collects more than the half of the light which is used for photosynthesis [1,2]. In 1994, Kühlbrandt and co-workers presented the first crystal structure of LHC II with a resolution of $3.4 \AA$ [3]. In this crystal structure 12 chlorophylls and 2 carotenoids were fully resolved. However, at this level of resolution it was not possible to determine which site was occupied by Chl $a$ or Chl $b$. In addition, no determination of the position of the phytyl-chains and thus of the direction of the transition dipole moments of $\mathrm{Q}_{y}$ and $\mathrm{Q}_{x}$ was possible. Without this information calculations of the energy transfer rates and potential, excitonic couplings between the pigments remained somewhat speculative [4-7].

\footnotetext{
* Corresponding author. Address: Max-Planck-Institute for Biophysical Chemistry, Department of Spectroscopy and Photochemical Kinetics Am Faßberg 11, D-37077 Göttingen, Germany.

E-mail address: pwalla@gwdg.de (P.J. Walla).
}

However, in 2004, Liu et al. published a new crystal structure of the LHC II trimer of spinach at a resolution of $2.72 \AA$ [8]. In this structure 14 chlorophylls per monomer could be resolved of which 8 are clearly assigned as Chl $a$ and 6 as $\mathrm{Chl} b$. A year later, Kühlbrandt and co-workers obtained a crystal structure of LHC II from pea at a resolution of $2.5 \AA$ [9]. Because of the better resolution in both structure models the orientation of all pigments could clearly be assigned.

Consequently, several groups have started to use the new structures for a much better theoretical description of the energy pathways in LHC II and to compare them with experimental results [10-20]. Novoderezhkin et al. simulated the spectroscopic properties of LHC II by using exciton theory [21]. To match the theoretical results with experimental data the diagonal elements of the Hamiltonian (site energies of the $\mathrm{Q}_{y}$ states) were varied while the off-diagonal elements (pigment-pigment couplings) were calculated using the ideal dipole approximation (IDA). This study [21], unravelled important details about the excitonic structure and important energy pathways in LHC II. However, the IDA usually breaks down for small distances between pigments because local interactions of the wave 
functions become important. An approach to overcome this problem has been reported by Linnanto et al. [22]. In this report [22], the site energies of the chlorophylls and the pigment-pigment couplings of those pairs with a distance of less than $15 \AA$ have been calculated using the semi-empirical ZINDO CIS method. Nevertheless, a corresponding calculation on the ab inito level would exceed the capacity of modern computational facilities. In this context the transition density cube (TDC) method has been developed by Krueger et al. which calculates coulombic interactions based on three-dimensional transition densities obtained from ab initio methods [23-25]. This approach yielded excellent agreement with experimental results for the bacterial light-harvesting complex LH 2 of Rps. acidophila $[23,26,27]$.

Here, we present a refined calculation of the inter-pigment couplings in LHC II based on the TDC method. For the calculation the high resolution crystal structure reported by Liu et al. was used [8]. A comparison of the couplings calculated by the TDC method with couplings calculated using the IDA demonstrate that this approximation cannot be used for pigment-pigment center to center distances of less than about $25 \AA$. In addition, we also calculated couplings involving the allowed second excited $\mathrm{S}_{2}$ state of the carotenoids from the new structure of LHC II and considered explicitly the $\mathrm{Q}_{x}$ band of the Chl molecules. These calculated couplings provide an improved basis for a future calculation of the energy path ways and exciton states including Cars and $\mathrm{Q}_{x}$.

\section{Theory and calculation}

Calculation of couplings by means of the IDA and TDC method have previously been described in detail [23-25]. Briefly, in the IDA the couplings, $V^{\mathrm{IDA}}$, are calculated according to the following equation

$V^{\mathrm{IDA}}=\frac{1}{\varepsilon_{\mathrm{r}}} \kappa \frac{\left|\vec{\mu}_{\mathrm{n}}\right|\left|\vec{\mu}_{\mathrm{m}}\right|}{R_{\mathrm{nm}}^{3}}$

Here, $\varepsilon_{r}$, is the relative dielectric constant, $R_{\mathrm{nm}}$, the distance between the pigments $\mathrm{n}$ and $\mathrm{m}$ and, $\kappa$, an orientation factor calculated from the angles, $\phi$, between the transition dipole moments $\vec{\mu}_{\mathrm{n}}, \vec{\mu}_{\mathrm{m}}$ and the connection vector $\vec{R}_{\mathrm{nm}}$

$$
\begin{gathered}
\kappa=\cos \left(\phi_{\mathrm{nm}}\right)-3\left(\cos \left(\phi_{\mathrm{n}}\right) \cos \left(\phi_{\mathrm{m}}\right)\right) \\
=\frac{\vec{\mu}_{\mathrm{n}} \vec{\mu}_{\mathrm{m}}}{\left|\vec{\mu}_{\mathrm{n}}\right|\left|\vec{\mu}_{\mathrm{m}}\right|}-3 \frac{\left(\vec{\mu}_{\mathrm{n}} \vec{R}_{\mathrm{nm}}\right)\left(\vec{\mu}_{\mathrm{m}} \vec{R}_{\mathrm{nm}}\right)}{\left|\vec{\mu}_{\mathrm{n}}\right|\left|\vec{\mu}_{\mathrm{m}}\right| R_{\mathrm{nm}}^{2}}
\end{gathered}
$$

In the IDA the orientation of the transition dipole moment vectors of chlorophyll molecules are often approximated by the axes along the nitrogens of the porphyrin structure, $\vec{\mu}_{\mathrm{N}-\mathrm{N}}$ (see e.g., [21] and Fig. 1). In the present Letter, we denote IDA couplings calculated in this way $V^{\mathrm{IDA}}(\mathrm{N}-\mathrm{N})$.

The exact coulombic coupling, $V^{\text {Coulomb }}$, can be described by the interaction of the transition densities of both molecules:

$V^{\text {Coulomb }}=\frac{1}{4 \pi \varepsilon_{0}} \iint \frac{M_{1}\left(r_{i}\right) M_{2}\left(r_{j}\right)}{r_{i j}} \mathrm{~d} r_{i} \mathrm{~d} r_{j}$

Here, $M(r)$ is the transition density $M(r)=\int \Psi_{\mathrm{g}}^{*} \Psi_{\mathrm{e}} \mathrm{d} s=$ $\left\langle\Psi_{\mathrm{g}} \mid \Psi_{\mathrm{e}}\right\rangle$ between the ground state and the excited state. Eq. (4) can be approximated by a summation over a grid of transition density cubes:

$V^{\mathrm{TDC}}=\frac{V_{1} \cdot V_{2}}{4 \pi \varepsilon_{0}} \sum_{i, j} \frac{M_{1, i} \cdot M_{2, j}}{r_{i j}}$

with

$M(x, y, z)=\int_{z}^{z+\delta z} \int_{y}^{y+\delta y} \int_{x}^{x+\delta x} \int \Psi_{\mathrm{g}}^{*} \Psi_{\mathrm{e}} \mathrm{d} s \mathrm{~d} x \mathrm{~d} y \mathrm{~d} z$

This is the TDC-method developed by Krueger et al. [2325]. Here, $V=\delta x \cdot \delta y \cdot \delta z$ is the volume of one cube and $r$ is the distance between two cubes. In the present Letter, the transitions were calculated with the HF-CIS method implemented in GAUSSIAN03 ${ }^{(\mathrm{R})}$ and mapped into cubes with the 'cube'-keyword. For each pigment about 300000 cubes with dimensions of $(0.3 \AA)^{3}$ were calculated. The resulting transition densities are scaled to match the magnitude of calculated transition dipole moments, $\left|\vec{\mu}_{\mathrm{TDC}}\right|$, with experimentally determined dipole moments, $\mu_{\text {exp. }}$ :

$$
\begin{aligned}
& M(x, y, z)_{\text {scaled }}=M(x, y, z) \frac{\mu_{\text {exp } .}}{\left|\vec{\mu}_{\mathrm{TDC}}\right|} \\
& \vec{\mu}_{\mathrm{TDC}}=V \cdot \sum_{M_{i} \vec{r}_{i}}
\end{aligned}
$$

The vector calculated from the $a b$ initio transition density cubes, $\vec{\mu}_{\text {TDC }}$, can also be used for a calculation of the couplings using the IDA (Eq. (1)). In the present Letter, we de-
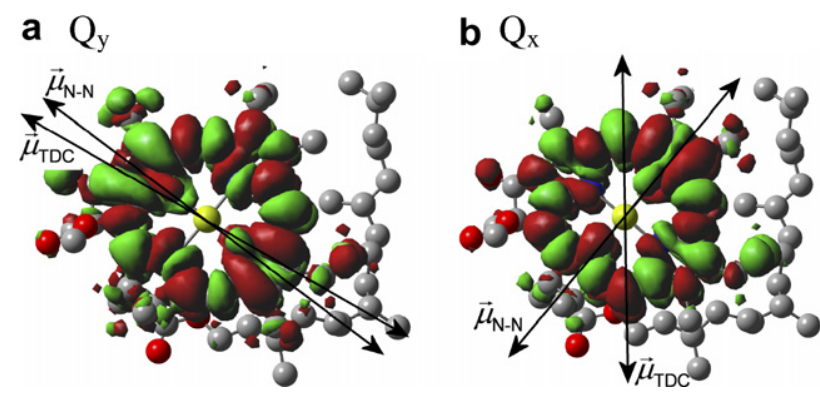

c $\mathrm{Car} \mathrm{S}_{0} \rightarrow \mathrm{Car} \mathrm{S}_{2}$

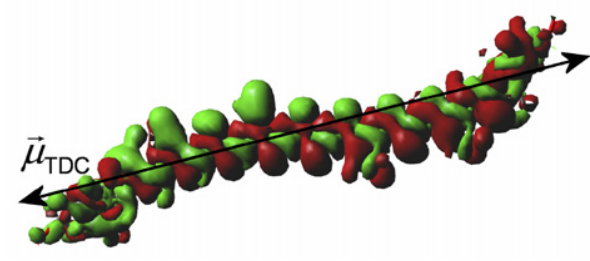

Fig. 1. Transition densities $M(x, y, z)$ and transition dipole moment vectors $\vec{\mu}_{\mathrm{N}-\mathrm{N}}$ and $\vec{\mu}_{\mathrm{TDC}}$, calculated for (a) the chlorophyll $\mathrm{Q}_{y}$ transition and (b) the chlorophyll $\mathrm{Q}_{x}$ transition of Chl $a 602$ and (c) the Car $\mathrm{S}_{0} \rightarrow$ Car $\mathrm{S}_{2}$ transition of Lut 620 . 
note IDA couplings calculated in this way as $V^{\mathrm{IDA}}(\mathrm{TDC})$. Especially for the Chl $\mathrm{Q}_{x}$ transition the direction of the vector of $\vec{\mu}_{\mathrm{TDC}}$ deviates significantly from $\vec{\mu}_{\mathrm{N}-\mathrm{N}}$ (Fig. 1).

For the final calculation of the exact couplings, $V^{\mathrm{TDC}}$, the following expression is used

$V^{\mathrm{TDC}}=\frac{1}{4 \pi \varepsilon_{0}} \frac{\mu_{1, \exp .} \cdot \mu_{2, \exp .}}{\left|\sum_{i} M_{i} \cdot \vec{r}_{i}\right| \cdot\left|\sum_{j} M_{j} \cdot \vec{r}_{j}\right|} \sum_{i, j} \frac{M_{1, i} \cdot M_{2, j}}{r_{i j}}$

For the scaling of the transition densities of the Chl $a \mathrm{Q}_{y}$ transitions we used the same dipole moments, $\mu_{\exp .}$, as Novoderezkin's et al. [21]. For the ratio between the dipole moments of Chl $b$ and $\mathrm{Chl} a$ Sauer et al. reported a value of $\sim 0.82$ [28]. For the ratio between the dipole moments of the $\mathrm{Q}_{x}$ and the $\mathrm{Q}_{y}$ transitions Damjanovic et al. reported a value of $\sim 0.67$ [29]. For the dipole moment for the carot-

Table 1

experimental transition dipole moment $\mu_{\text {exp. }}$ used for scalling

\begin{tabular}{lc}
\hline Chl $a \mathrm{Q}_{y}$ & $3.74 \mathrm{D}$ \\
$\mathrm{Chl} a \mathrm{Q}_{x}$ & $3.07 \mathrm{D}$ \\
$\mathrm{Chl} b \mathrm{Q}_{y}$ & $3.19 \mathrm{D}$ \\
$\mathrm{Chl} b \mathrm{Q}_{x}$ & $2.61 \mathrm{D}$ \\
Car $\mathrm{S}_{2}$ & $13.5 \mathrm{D}$ \\
\hline
\end{tabular}
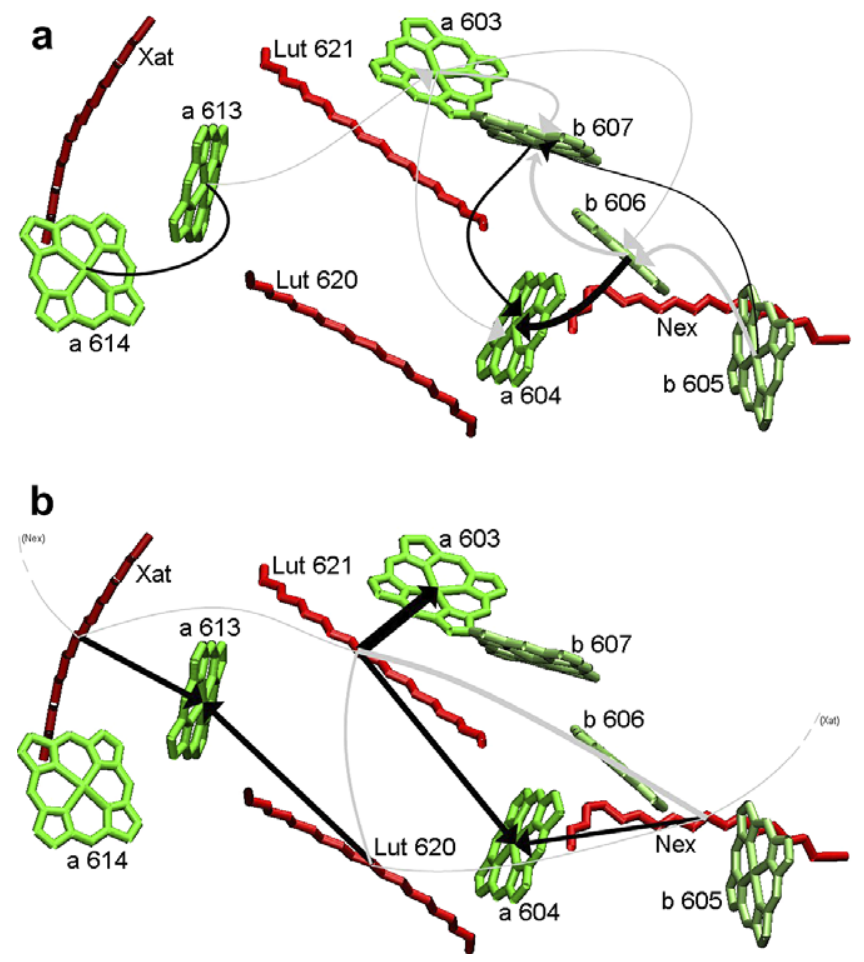

Fig. 2. Arrangement of the chlorophylls ( $a$ : light green, $b$ : dark green) and carotenoids (red) at the lumenal side of LHC II. (a) Chlorophyllchlorophyll couplings. Only the biggest couplings between the Chl-pairs are shown. The width of the connecting lines scales linearly with its magnitude. (- $\mathrm{Q}_{y}-\mathrm{Q}_{y}$ coupling between two Chl's $a$ or Chl's $b ;(\rightarrow)$ Chl $b \mathrm{Q}_{y}$-Chl $a \mathrm{Q}_{y}$ coupling; $(\longrightarrow) \mathrm{Chl} a / b \mathrm{Q}_{x}$-Chl $a / b \mathrm{Q}_{y}$ coupling. (b) Carotenoid $\mathrm{S}_{2}$-Chlorophyll couplings. $(\rightarrow)$ Car $\mathrm{S}_{2}-\mathrm{Chl}$ coupling; $(-)$ Car $\mathrm{S}_{2}-$ Car $\mathrm{S}_{2}$ coupling. enoid's $S_{0} \leftarrow S_{2}$ transition we estimated a similar value as Gradinaru et al. [30] based on experimental absorption spectra of the LHC II carotenoids in solution. The experimental transition dipole moments, $\mu_{\text {exp. }}$, used for the present calculations are summarized in Table 1. The coupling constants presented in Section 3 can simply be scaled linearly with any other experimentally observed transition dipole moment, $\mu_{\text {exp. }}$ (Eq. (7)) [31].

\section{Results}

The pigments in LHC II can be divided into a stromal and lumenal part. In the following tables and figures assignments of the pigments where used as suggested by Liu et al. [8].

The strongest and most important couplings in the lumenal and stromal side of LHC II are visualized in Figs. 2 and 3, respectively. The corresponding numeric values are presented in Tables 2-5 including those couplings which are not visualized in Figs. 2 and 3. In Figs $2 \mathrm{a}$ and $3 \mathrm{a}$ black lines without arrows indicate strong $\mathrm{Q}_{y}-\mathrm{Q}_{y}$ couplings between pairs of Chl $a$ or Chl $b$ molecules (Table 2), which could result in strong exciton couplings when the lowest $\mathrm{Q}_{y}$-states have similar site energies in the monomeric site energy presentation. In general, the thickness of the lines represents the magnitude of the calculated couplings. Black arrows represent strong cou-
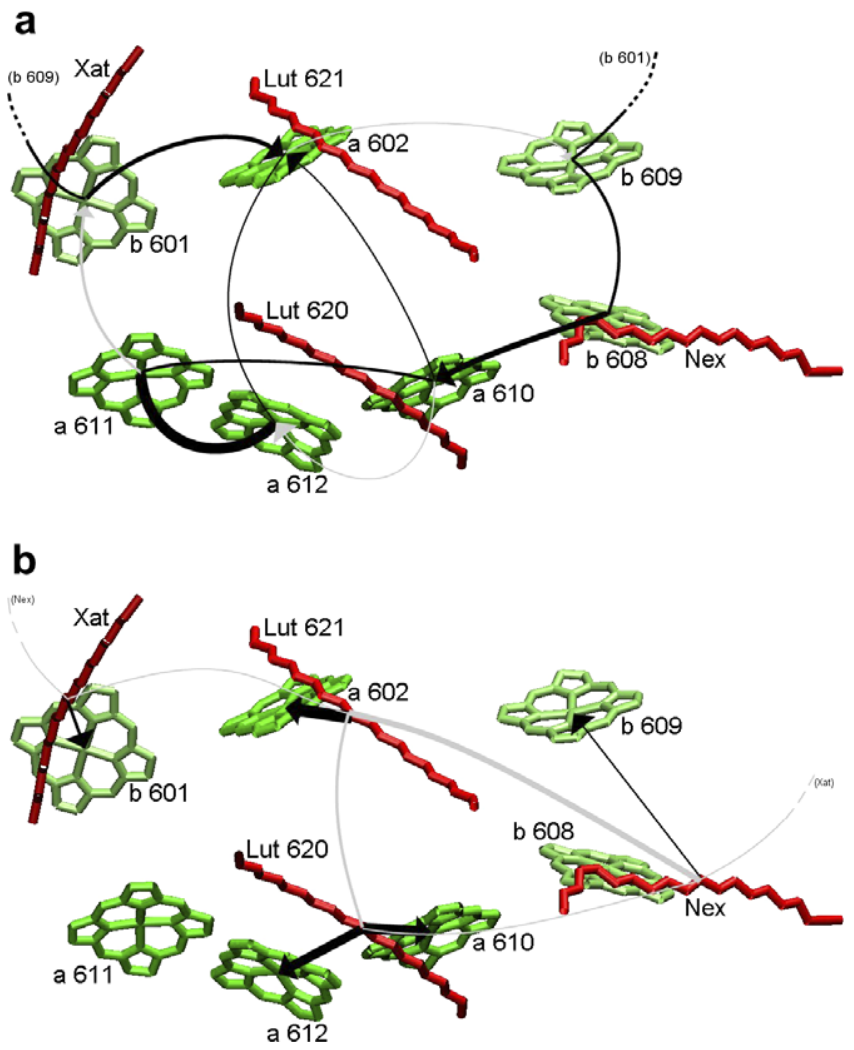

Fig. 3. Arrangement of the chlorophylls at the stromal side of LHC II. Lines and arrows have the same meaning as in Fig. 2. 
Table 2

Couplings, $V^{\mathrm{TDC}}$, between $\mathrm{Q}_{y}$ and $\mathrm{Q}_{x}$-transitions between pairs of chlorophylls at the stromal side and lumenal side of LHC II

\begin{tabular}{lllllllll}
$\frac{a 602}{y}$ & $\frac{a 610}{y}$ & $\frac{a 611}{y}$ & $\frac{a 612}{y}$ & $\frac{b 601}{y}$ & $x$ & $x$ \\
\hline
\end{tabular}

Stromal chlorophyll-chlorophyll couplings $\left(\mathrm{cm}^{-1}\right)$

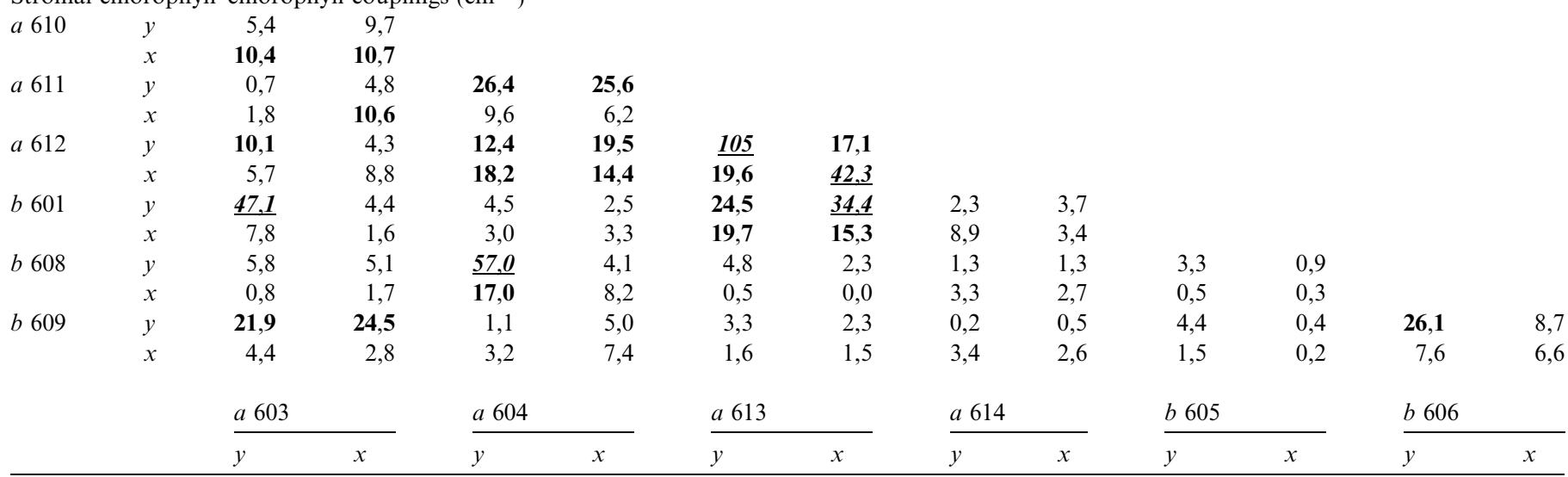

Lumenal chlorophyll-chlorophyll couplings $\left(\mathrm{cm}^{-1}\right)$

$\begin{array}{llll}a 604 & y & 0,5 & \mathbf{1 9 , 9} \\ & x & 1,1 & \mathbf{2 5 , 5}\end{array}$

$\begin{array}{llrr}a & 613 & y & 2,4\end{array}$

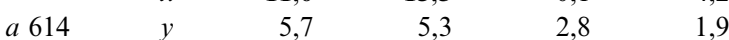

$\begin{array}{lllll}x & 2,9 & 1,2 & 0,9 & 1,8\end{array}$

$\begin{array}{llllll}b 605 & y & 0,2 & 0,9 & 5,4 & 2,5\end{array}$

$\begin{array}{lll}x & 1,1 & 2,9\end{array}$

$\begin{array}{llll}b 606 & y & 2,1 & \mathbf{2 0 , 8}\end{array}$

$3,9 \quad 6,2$

$\underline{80,8} \quad \underline{53,0}$

$3,7 \quad 21,4 \quad \underline{62,6} \quad \underline{16,9}$

$\begin{array}{llllll}b 607 & y & 8,2 & \underline{\mathbf{3 8 , 2}} & \underline{\mathbf{2 6 , 0}} & \mathbf{1 6 , 6}\end{array}$

$16,9 \quad \underline{34,5} \quad 1,0 \quad 2,9$

$\mathbf{2 8 , 0}$
9,8
1,2
0,2
1,2
2,4
2,7
1,1

$\begin{array}{rrr}\mathbf{1 5 , 9} & & \\ \mathbf{1 8 , 9} & & \\ 1,0 & 0,0 & 0,5 \\ 0,1 & 0,7 & 0,7 \\ 0,4 & 1,8 & 1,3 \\ 0,9 & 0,9 & 0,4 \\ 1,2 & 2,4 & 1,7 \\ 3,3 & 2,7 & 1,9\end{array}$

\begin{tabular}{rrrrr}
0,5 & & & & \\
0,7 & & & & \\
1,3 & $\mathbf{1 1 , 5}$ & $\underline{\mathbf{4 0 , 1}}$ & & \\
0,4 & 4,3 & 9,0 & & \\
1,7 & 5,2 & 0,5 & $\mathbf{2 3 , 7}$ & $\underline{\mathbf{5 1 , 5}}$ \\
1,9 & 1,3 & $\mathbf{1 0 , 1}$ & $\mathbf{1 7 , 6}$ & $\mathbf{1 , 6}$ \\
\hline
\end{tabular}

Values $>10 \mathrm{~cm}^{-1}$ are marked bold. The strongest couplings $\left(>30 \mathrm{~cm}^{-1}\right)$ are underlined and marked bold and italic.

plings between $\mathrm{Chl} b$ and $\mathrm{Chl} a$ pigments. The arrows point towards the $\mathrm{Chl} a$ pigment to indicate the direction of a potential energy path way. Similarly, grey arrows represent strong $\mathrm{Q}_{x}$ and $\mathrm{Q}_{y}$ couplings between any $\mathrm{Chl}$ pigment. In Figs. $2 \mathrm{~b}$ and $3 \mathrm{~b}$ the strongest inter-pigment couplings of the carotenoid states, Car $\mathrm{S}_{2}$, and chlorophylls in the lumenal side and stromal side are visualized, respectively. Black arrows indicate strong carotenoid to chlorophyll couplings, potentially resulting in effective Car $\mathrm{S}_{2} \rightarrow$ Chl energy transfer. Grey lines indicate strong Car $\mathrm{S}_{2}-$ Car $\mathrm{S}_{2}$ couplings, potentially resulting in excitonic Car $\mathrm{S}_{2}$ states.

Fig. 4 shows the ratio between couplings calculated by the TDC method, $V^{\mathrm{TDC}}$, and the IDA, $V^{\mathrm{IDA}}(\mathrm{TDC})$, of Chl-Chl couplings (black dots) and Car-Chl couplings (red dots) as a function of the inter-pigment distance. It is obvious that the IDA is drastically deviating for distances smaller than about $25 \AA$. Large couplings are often observed for small inter-pigment distances. Therefore, it is not surprising that for large couplings quite large differences can sometimes be obtained. In Table 6 the values for the 10 biggest couplings as calculated by the IDA are shown, as examples, in comparison with the corresponding values calculated from the TDC-method.

\section{Discussion}

The most important result of the present Letter is a dramatically increasing deviation of the couplings calculated by the IDA from the TDC-couplings for smaller inter-pigment distances (Fig. 4). In general, for distances smaller than $25 \AA$ the IDA yields results differing by up to one order of magnitude from the TDC calculation. While the strongest $\mathrm{Chl}_{y}-\mathrm{Chl} \mathrm{Q}_{y}$ couplings are often described still reasonable well by the IDA, strong couplings involving $\mathrm{Chl}$ $\mathrm{Q}_{x}$ transitions show, in many cases, large deviations from the exact TDC-values (Fig. 4 and examples in Table 6). For example, TDC calculations find a significantly weaker coupling between the Chl $a 612 \mathrm{Q}_{x}$ transition and the Chl $a$ $610 \mathrm{Q}_{y}$ transition $\left(V^{\mathrm{TDC}}=18 \mathrm{~cm}^{-1}\right)$ than the IDA $\left(V^{\mathrm{IDA}}(\mathrm{N}-\mathrm{N})=52 \mathrm{~cm}^{-1}, V^{\mathrm{IDA}}(\mathrm{TDC})=35 \mathrm{~cm}^{-1}\right)$. The main reason for this is that the $\mathrm{Q}_{x}$ transition has a considerable multipole character, which explains the large deviations often observed between $V^{\mathrm{TDA}}$ (TDC) and $V^{\mathrm{TDC}}$ for interactions in which these states are involved (Fig. 1). In addition, the orientation of dipole-moment vector of the $\mathrm{Q}_{x^{-}}$ transitions calculated by the $a b$ initio transition densities, $\vec{\mu}_{\mathrm{TDC}}$, derivates by about $40^{\circ}$ from a dipole moment $\vec{\mu}_{\mathrm{N}-\mathrm{N}}$ along the nitrogen-nitrogen axis in the porphyrin ring 
Table 3

Inter-stromal-lumenal (top) and inter-monomeric (bottom) chlorophyll couplings, $V^{\mathrm{TDC}}$

\begin{tabular}{|c|c|c|c|c|c|c|c|c|c|c|c|c|c|c|c|}
\hline & & \multicolumn{2}{|l|}{$a 602$} & \multicolumn{2}{|c|}{$a 610$} & \multicolumn{2}{|c|}{ a 611} & \multicolumn{2}{|l|}{$a 612$} & \multicolumn{2}{|c|}{$b 601$} & \multicolumn{2}{|c|}{$b 608$} & \multicolumn{2}{|l|}{$b 609$} \\
\hline & & $y$ & $x$ & $y$ & $x$ & $y$ & $x$ & $y$ & $x$ & $y$ & $x$ & $y$ & $x$ & $y$ & $x$ \\
\hline \multicolumn{16}{|c|}{ Inter-stromal-lumenal chlorophyll-chlorophyll couplings $\left(\mathrm{cm}^{-1}\right)$} \\
\hline \multirow[t]{2}{*}{ a 603} & $y$ & 17,4 & 25,5 & 8,4 & 3,8 & 0,7 & 4,2 & 0,6 & 2,4 & 6,1 & 1,4 & 4,2 & 2,3 & $\underline{71,6}$ & 7,2 \\
\hline & $x$ & 19,7 & 16,9 & 3,3 & 10,9 & 1,4 & 2,7 & 4,5 & 6,4 & 4,9 & 1,9 & 3,8 & 1,7 & 2,6 & 27,5 \\
\hline \multirow[t]{2}{*}{$a 604$} & $y$ & 5,5 & 3,9 & 0,2 & 18,1 & 3,3 & 1,9 & 3,7 & 3,3 & 2,7 & 0,2 & 5,7 & 5,1 & 1,5 & 24,0 \\
\hline & $x$ & 6,7 & 6,8 & 8,6 & 19,3 & 0,9 & 0,1 & 20,2 & 24,6 & 1,4 & 0,6 & 5,7 & 4,8 & 2,2 & 28,3 \\
\hline \multirow[t]{2}{*}{$a 613$} & $y$ & 1,9 & 12,8 & 6,0 & 4,3 & 0,8 & 26,5 & 1,0 & 20,5 & 8,4 & 3,4 & 2,2 & 0,1 & 2,5 & 1,0 \\
\hline & $x$ & 6,7 & 5,6 & 6,6 & 6,1 & 0,1 & $\underline{38,3}$ & 0,4 & 27,0 & 5,9 & 6,4 & 1,1 & 0,1 & 0,1 & 0,0 \\
\hline \multirow[t]{2}{*}{$a 614$} & $y$ & 0,1 & 7,0 & 1,2 & 0,0 & 0,6 & 15,0 & 0,2 & 3,6 & 2,9 & 4,0 & 1,4 & 0,2 & 2,0 & 1,0 \\
\hline & $x$ & 0,8 & 2,9 & 3,0 & 1,3 & 3,6 & 13,0 & 4,6 & 12,3 & 3,5 & 0,2 & 1,1 & 0,4 & 1,2 & 1,4 \\
\hline \multirow[t]{2}{*}{ b 605} & $y$ & 0,2 & 0,0 & 0,8 & 2,2 & 1,1 & 0,4 & 2,2 & 2,4 & 0,5 & 0,4 & 3,7 & 4,5 & 0,1 & 1,2 \\
\hline & $x$ & 1,3 & 0,9 & 0,8 & 2,2 & 0,8 & 0,6 & 1,2 & 1,1 & 0,7 & 0,1 & 1,6 & 2,6 & 3,5 & 2,3 \\
\hline \multirow[t]{2}{*}{ b 606} & $y$ & 4,9 & 3,9 & 0,6 & 8,9 & 2,0 & 1,6 & 2,1 & 1,5 & 2,0 & 0,1 & 6,7 & 2,5 & 11,8 & $\underline{53,1}$ \\
\hline & $x$ & 4,0 & 3,9 & 2,3 & 9,5 & 2,1 & 0,9 & 1,9 & 1,9 & 1,6 & 0,4 & 0,6 & 6,7 & 12,3 & 46,2 \\
\hline \multirow[t]{4}{*}{$b 607$} & $y$ & 6,2 & 4,7 & 0,4 & 4,6 & 2,1 & 2,0 & 2,2 & 1,3 & 2,6 & 0,1 & 3,5 & 0,6 & 1,7 & $\overline{18,8}$ \\
\hline & $x$ & 4,0 & 1,8 & 2,9 & 0,2 & 0,7 & 1,8 & 0,9 & 0,5 & 1,7 & 0,4 & 5,3 & 3,7 & 16,0 & 7,5 \\
\hline & & \multicolumn{2}{|l|}{ a 602} & \multicolumn{2}{|c|}{ a 603} & \multicolumn{2}{|c|}{ a 611} & \multicolumn{2}{|l|}{ a 613} & \multicolumn{2}{|c|}{ a 614} & \multicolumn{2}{|c|}{$b 601$} & & \\
\hline & & $y$ & $x$ & $y$ & $x$ & $y$ & $x$ & $y$ & $x$ & $y$ & $x$ & $y$ & $x$ & & \\
\hline \multicolumn{16}{|c|}{ Significant inter-monomer couplings $\left(\mathrm{cm}^{-1}\right)$} \\
\hline \multirow[t]{2}{*}{$b 606$} & $y$ & & & & & & & & & 5,0 & & & & & \\
\hline & $x$ & & & & & & & 5,3 & & & & & & & \\
\hline \multirow[t]{2}{*}{ b 607} & $y$ & & & & & & & 9,8 & & & & & & & \\
\hline & $x$ & & & & & & & & & 5,1 & & & & & \\
\hline \multirow[t]{2}{*}{$b 608$} & $y$ & & & & & & & & & & & 6,0 & & & \\
\hline & $x$ & & & & & & & & & & & & & & \\
\hline$b 609$ & $y$ & 6,8 & 6,1 & & & & & & & & & $\underline{35,7}$ & 27,5 & & \\
\hline & $x$ & 9,4 & 5,1 & & & & & 6,6 & & & & 22,0 & 22,7 & & \\
\hline Lut 620 & & 5,7 & & & & & & & & & & 6,8 & & & \\
\hline Lut 621 & & 9,4 & 13,4 & 8,8 & & & & 6,5 & & 7,5 & & 9,2 & 10,4 & & \\
\hline Xat 622 & & & & & & & & & & & & & & & \\
\hline Nex 623 & & 7,0 & & & & 8,8 & 10,3 & & 9,0 & 8,7 & 5,9 & & 19,3 & & \\
\hline
\end{tabular}

Stronger couplings are marked in the same way as in Table 2. Only inter-monomeric couplings which are larger than $5 \mathrm{~cm}^{-1}$ are shown.

Table 4

Couplings, $V^{\mathrm{TDC}}$, between the $\mathrm{S}_{2}$ state of carotenoids and $\mathrm{Q}_{y}$ and $\mathrm{Q}_{x}$-transitions of chlorophylls in the stromal and lumenal side

\begin{tabular}{|c|c|c|c|c|c|c|c|c|c|c|c|c|c|c|}
\hline & \multicolumn{2}{|l|}{$a 602$} & \multicolumn{2}{|l|}{ a 610} & \multicolumn{2}{|l|}{ a 611} & \multicolumn{2}{|c|}{$a 612$} & \multicolumn{2}{|c|}{$b 601$} & \multicolumn{2}{|c|}{ b608 } & \multicolumn{2}{|l|}{$b 609$} \\
\hline & $y$ & $x$ & $y$ & $x$ & $y$ & $x$ & $y$ & $x$ & $y$ & $x$ & $y$ & $x$ & $y$ & $x$ \\
\hline \multicolumn{15}{|c|}{ Stromal carotenoid $\mathrm{S}_{2}$-chlorophyll couplings $\left(\mathrm{cm}^{-1}\right)$} \\
\hline Lut 620 & 4,6 & 39,9 & 34,1 & $\underline{208,3}$ & 8,7 & 43,9 & 5,0 & $\underline{190,5}$ & 14,7 & 0,3 & 14,4 & 13,1 & 4,4 & 2,4 \\
\hline Lut 621 & 17,0 & $\underline{221,2}$ & 3,7 & 46,2 & 6,2 & 10,2 & 32,4 & 34,7 & 45,8 & 8,8 & 6,9 & 13,1 & 8,9 & 60,0 \\
\hline Xat 622 & 33,6 & $\overline{20,0}$ & 7,4 & 9,3 & 49,4 & 18,1 & 23,1 & 13,7 & 95,6 & 36,9 & 3,5 & 2,0 & 3,7 & 1,3 \\
\hline \multirow[t]{3}{*}{ Nex 623} & 6,9 & 1,2 & 12,0 & 40,7 & 7,5 & 5,6 & 21,6 & 19,4 & 4,8 & 0,9 & 33,2 & 20,9 & 9,0 & 65,8 \\
\hline & \multicolumn{2}{|l|}{$a 603$} & \multicolumn{2}{|l|}{$a 604$} & \multicolumn{2}{|l|}{ a 613} & \multicolumn{2}{|c|}{$a 614$} & \multicolumn{2}{|c|}{ b 605} & \multicolumn{2}{|c|}{$b 606$} & \multicolumn{2}{|c|}{$b 607$} \\
\hline & $y$ & $x$ & $y$ & $x$ & $y$ & $x$ & $y$ & $x$ & $y$ & $x$ & $y$ & $x$ & $y$ & $x$ \\
\hline \multicolumn{15}{|c|}{ Lumenal carotenoid $\mathrm{S}_{2}$-chlorophyll couplings $\left(\mathrm{cm}^{-1}\right)$} \\
\hline Lut 620 & 28,2 & 27,8 & 18,5 & 31,0 & $\underline{136,2}$ & $\underline{140,1}$ & 31,7 & 60,0 & 10,7 & 3,0 & 2,5 & 13,6 & 1,6 & 15,0 \\
\hline Lut 621 & 44,2 & $\underline{296,9}$ & $\underline{133,6}$ & 149,9 & 9,9 & $\overline{28,1}$ & 16,2 & 1,5 & 5,4 & 11,6 & 71,5 & 59,5 & 72,5 & 56,1 \\
\hline Xat 622 & 3,9 & 2,4 & 1,7 & 1,3 & 127,7 & 49,3 & 52,4 & 38,9 & 1,7 & 0,3 & 0,0 & 3,6 & 1,5 & 5,3 \\
\hline Nex 623 & 7,0 & 23,1 & $\underline{105,5}$ & 101,1 & 4,8 & 2,9 & 2,6 & 6,0 & 22,9 & 15,2 & 64,2 & 79,1 & 17,7 & 13,5 \\
\hline
\end{tabular}

Values $>50 \mathrm{~cm}^{-1}$ are marked bold. The strongest couplings $\left(>100 \mathrm{~cm}^{-1}\right)$ are underlined and marked bold and italic.

structure. This explains that for $\mathrm{Q}_{x}$-transitions even larger deviations are observed between $V^{\mathrm{IDA}}(\mathrm{N}-\mathrm{N})$ and $V^{\mathrm{TDC}}$. Consequently, a detailed TDC-calculation is at least necessary for an exact description of energy-transfer path ways in which the $\mathrm{Q}_{x}$ states are often strongly involved.
The calculated couplings in the present Letter confirm that the strongest potential exciton interactions between the lowest $\mathrm{Q}_{y}$ states occur between the pigments around $\mathrm{Chl} a 611$ and Chl $a 612$ in the stromal side [21]. Interestingly, there is also a significant $\mathrm{Q}_{y}-\mathrm{Q}_{y}$ coupling between 
Table 5

Couplings, $V^{\mathrm{TDC}}$, between the carotenoids within a monomer (upper part) and with carotenoids of the neighbouring monomer (lower part)

\begin{tabular}{cccc}
\hline Carotenoid $\mathrm{S}_{2}$-carotenoid $\mathrm{S}_{2}$ couplings $\left(\mathrm{cm}^{-1}\right)$ & & \\
\hline Lut 620 & Lut 621 & Xat 622 & Nex 623
\end{tabular}

\begin{tabular}{lrrrr}
\hline Lut 621 & $\mathbf{8 1 , 5}$ & & & \\
Xat 622 & 2,2 & $\mathbf{5 0 , 4}$ & & \\
Nex 623 & $\mathbf{5 7 , 3}$ & $\underline{\mathbf{1 2 9 , 0}}$ & 2,9 & \\
Lut 620 & 1,8 & 6,4 & 22,9 & 0,4 \\
Lut 621 & 16,2 & 9,3 & 29,1 & 3,9 \\
Xat 622 & 4,5 & 7,0 & 18,5 & 4,8 \\
Nex 623 & 3,2 & 6,3 & $\mathbf{6 6 , 7}$ & 1,2 \\
\hline
\end{tabular}

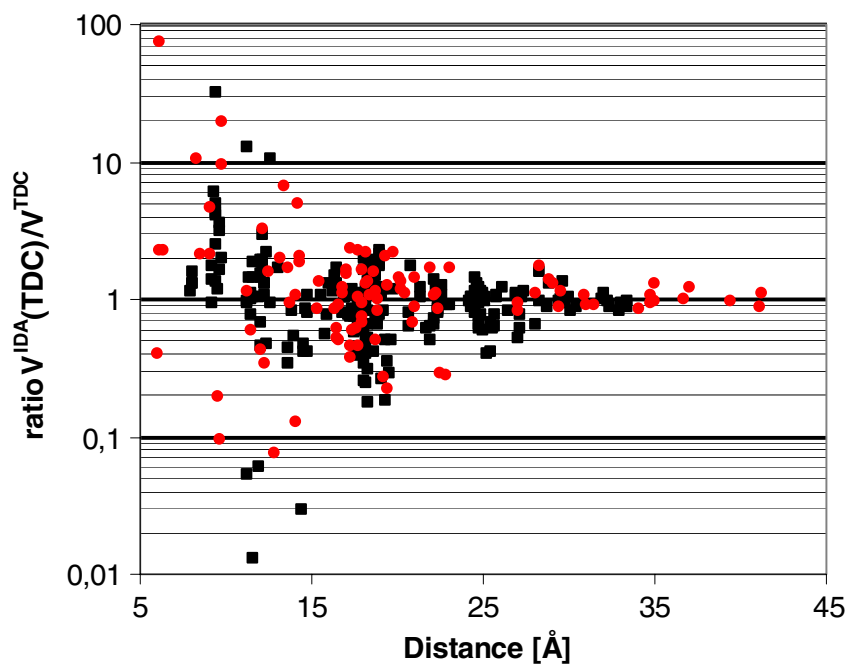

Fig. 4. Ratio of couplings calculated by the IDA ( $V^{\mathrm{IDA}}$ (TDC), Eqs. (1) and (6)) and the TDC-method ( $V^{\mathrm{TDC}}$, Eq. (7)) as a function of the interpigment distance. Black dots represent Chl-Chl couplings and red dots Car-Chl couplings. The inter-pigment distance was calculated from the centers of weight of the transition densities which were close the Mg-atoms in the case of chlorophyll molecules. Only couplings larger than $2 \mathrm{~cm}^{-1}$ are shown.

Chl $a 602$ and Chl $a 612$ even though they are located quite far apart from each other (Fig. 3a and Table 2, top). This coupling is caused by a close-to-parallel alignment of the corresponding dipole-vectors resulting in a comparatively large value of the orientational factor $\kappa$. In the lumenal side the strongest $\mathrm{Q}_{y}-\mathrm{Q}_{y}$ coupling occurs between $\mathrm{Chl} a 614$ and Chl $a 613$ (Fig. 2a and Table 2, bottom). There are also significant inter-monomeric $\mathrm{Q}_{y}-\mathrm{Q}_{y}$ couplings present, for example between $\mathrm{Chl} b 601$ and $\mathrm{Chl} b 609$ on the stromal side (Table 3 , bottom).

The strongest $\mathrm{Chl} b-\mathrm{Chl} a$ and $\mathrm{Q}_{x}-\mathrm{Q}_{y}$ couplings give some hint with regard to preferred general energy pathways. An accumulation of potential energy pathways is present for Chl $a 604$ in the lumenal side as acceptor (Fig. 2, three strong Chl $b$ or $\mathrm{Chl} \mathrm{Q}_{x}$ to Chl $a 604$ couplings and two strong Car $\mathrm{S}_{2}$ to $\mathrm{Chl} a 604$ couplings). This result is in agreement with a large primary population of Chl $a$ 604 as predicted by Novoderezhkin et al. [21]. It is interesting that the largest potential energy transfer couplings are with the Chl $a 604$ (Fig. 2) molecules as acceptors, which have been assigned by Novoderezhkin et al. to be rather high in energy. Chl $a 604$ in the lumenal side is remote from Chl $a 612$ and its surrounding $\mathrm{Chl}$ molecules in the stromal side (Fig. 3) which has been predicted [21] to form the deepest trap in LHC II. This might indicate that it is advantageous for the major light-harvesting pigment pool to distribute the energy over the antenna first, rather then directing it directly to an energetic trap. As expected, carotenoids have the largest couplings with neighbouring $\mathrm{Chl}$ molecules (Figs. 2b and 3b; Table 5), even though there are exceptions (e.g., Nex-Chl $b 608$ (Fig. 3b), Lut 620-Chl $a 604$ (Fig. 2b), Nex-Chl $b 606$ (Fig. 2b)).

In summary, the present Letter demonstrates that great care has to be taken when using the IDA for the calculation of inter-pigment interactions in densely packed pigmentprotein complexes. The IDA often fails for small inter-pigment distances especially when chlorophyll $\mathrm{Q}_{x}$-transition are involved. In this context, the TDC-couplings presented in this Letter will provide a solid basis for future calculation of excitonic interactions and energy pathways in LHC II.

\section{Acknowledgements}

We thank Prof. B.P. Krueger for providing us with important information concerning the computational implementation of the TDC-method. We thank I. Dreger for helping with the preparation of the manuscript. This

Table 6

Comparison of the 10 biggest couplings as calculated by the IDA, $V^{\mathrm{IDA}}(\mathrm{N}-\mathrm{N})$ and $V^{\mathrm{IDA}}$ (TDC), with couplings calculated by the TDC-method according to Eq. (7), $V^{\text {TDC }}$

\begin{tabular}{|c|c|c|c|c|c|c|}
\hline Pigments involved & $\begin{array}{l}V^{\mathrm{IDA}}(\mathrm{N}-\mathrm{N}) \\
\left(\mathrm{cm}^{-1}\right)\end{array}$ & $\begin{array}{l}V^{\mathrm{IDA}}(\mathrm{TDC}) \\
\left(\mathrm{cm}^{-1}\right)\end{array}$ & $\begin{array}{l}V^{\mathrm{TDC}} \\
\left(\mathrm{cm}^{-1}\right)\end{array}$ & $\begin{array}{l}\text { Inter-pigment } \\
\text { distance }(\AA)\end{array}$ & $V^{\mathrm{IDA}}(\mathrm{N}-\mathrm{N}) / V^{\mathrm{TDC}}$ & $V^{\mathrm{IDA}}(\mathrm{TDC}) / V^{\mathrm{TDC}}$ \\
\hline Chl $611 a \mathrm{Q}_{y}-\mathrm{Chl} 612 a \mathrm{Q}_{y}$ & 114 & 122 & 105 & 9.5 & 1.1 & 1.2 \\
\hline Chl $604 a \mathrm{Q}_{y}-\mathrm{Chl} 606 b \mathrm{Q}_{y}$ & 95 & 106 & 81 & 8.0 & 1.2 & 1.3 \\
\hline Chl $603 a \mathrm{Q}_{y}-\mathrm{Chl} 609 b \mathrm{Q}_{y}$ & 84 & 95 & 72 & 9.4 & 1.2 & 1.3 \\
\hline Chl $604 a \mathrm{Q}_{x}-\mathrm{Chl} 606 b \mathrm{Q}_{x}$ & 71 & 27 & 17 & 8.0 & 4.2 & 1.6 \\
\hline Chl $606 b \mathrm{Q}_{x}-\mathrm{Chl} 607 b \mathrm{Q}_{x}$ & 59.8 & 30.5 & 1.64 & 8.9 & 36.5 & 18.6 \\
\hline Chl $610 a \mathrm{Q}_{y}-\mathrm{Chl} 612 a \mathrm{Q}_{x}$ & 52 & 35 & 18 & 12.1 & 2.9 & 1.9 \\
\hline Chl $606 b \mathrm{Q}_{y}-\mathrm{Chl} 607 b \mathrm{Q}_{y}$ & 51 & 59 & 24 & 9.4 & 2.1 & 2.5 \\
\hline
\end{tabular}


work is financially supported by a generous grant from the Fonds der chemischen Industrie and the Juniorstart-Programm of the Federal Ministry of Research and Education.

\section{References}

[1] S. Jansson, Biochim. Biophys. Acta-Bioenergetics 1184 (1994) 1.

[2] G.F. Peter, O. Mackold, J.P. Thornber, in: L.J. Rogers (Ed.), Methods in Plant Biochemistry, Academic Press, New York, 1988.

[3] W. Kühlbrandt, D.N. Wang, Y. Fujiyoshi, Nature 367 (1994) 614.

[4] V. Novoderezhkin, M. Palacios, H. van Amerongen, R. van Grondelle, J. Phys. Chem. B 108 (2004) 10363.

[5] T. Renger, V. May, Phys. Rev. Lett. 84 (2000) 5228.

[6] E.I. Iseri, D. Gülen, Eur. Biophys. J. 30 (2001) 344.

[7] V. Novoderezhkin, J.M. Salverda, H. van Amerongen, R. van Grondelle, J. Phys. Chem. B 107 (2003) 1893.

[8] Z. Liu, H. Yan, K. Wang, T. Kuang, J. Zhang, L. Gui, X. An, W. Chang, Nature 428 (2004) 287.

[9] J. Standfuss, A.C.T. van Scheltinga, M. Lamborghini, W. Kühlbrandt, EMBO J. 24 (2005) 919.

[10] P.J. Walla, J. Yom, B.P. Krueger, G.R. Fleming, J. Phys. Chem. B 104 (2000) 4799.

[11] R. Agarwal, B.P. Krueger, G.D. Scholes, M. Yang, J. Yom, L. Mets, G.R. Fleming, J. Phys. Chem. B 104 (2000) 2098.

[12] A. Wehling, P.J. Walla, J. Phys. Chem. B 109 (2005) 24510.

[13] T. Bittner, G.P. Wiederrecht, K.-D. Irrgang, G. Renger, M.R. Wasielewski, Chem. Phys. 194 (1995) 311.

[14] J.P. Connelly et al., J. Phys. Chem. B 101 (1997) 1902.

[15] M. Du, X. Xie, L. Mets, G.R. Fleming, J. Phys. Chem. 98 (1994) 4736
[16] C.C. Gradinaru, S. Özdemir, D. Gülen, I.H.M. van Stockkum, R. van Grondelle, H. van Amerongen, Biophys. J 75 (1998) 3064.

[17] C.C. Gradinaru, I.H.M. van Stockkum, A.A. Pascal, R. van Grondelle, H. van Amerongen, J. Phys. Chem. B (2000) 104.

[18] F.J. Kleima, C.C. Gradinaru, F. Calkoen, I.H.M. van Stockkum, R. van Grondelle, H. van Amerongen, Biochemistry 36 (1997) 15262.

[19] J.M. Salverda et al., Biophys. J. 84 (2003) 450.

[20] H.M. Visser, F.J. Kleima, I.H.M. van Stockkum, R. van Grondelle, H. van Amerongen, J. Chem. Phys. 210 (1996) 297.

[21] V.I. Novoderezhkin, M.A. Palacios, H.v. Amerongen, R.v. Grondelle, J. Phys. Chem. B 109 (2005) 10493.

[22] J. Linnanto, J. Martiskainen, V. Lehtovuori, J. Ihalainen, R. Kananavicius, R. Barbato, J. Korppi-Tommola, Photosynth. Res. 87 (2006) 267.

[23] B.P. Krueger, G.D. Scholes, R. Jimenez, G.R. Fleming, J. Phys. Chem. B 102 (1998) 2284.

[24] B.P. Krueger, G.D. Scholes, G.R. Fleming, J. Phys. Chem. B 102 (1998) 5378.

[25] B.P. Krueger, G.D. Scholes, G.R. Fleming, J. Phys. Chem. B 102 (1998) 9603.

[26] P.J. Walla, P.A. Linden, C.-P. Hsu, G.D. Scholes, G.R. Fleming, PNAS 97 (2000) 10808.

[27] C.-P. Hsu, P.J. Walla, M. Head-Gordon, G.R. Fleming, J. Phys. Chem. B 105 (2001) 11016.

[28] K. Sauer, J.R.L. Smith, A.J. Schultz, J. Am. Chem. Soc. 88 (1966) 2681.

[29] A. Damjanovic, T. Ritz, K. Schulten, Biophys. J. 79 (2000) 1695.

[30] C.C. Gradinaru, I.H.M. van Stokkum, A.A. Pascal, R. van Grondelle, H. van Amerongen, J. Phys. Chem. B 104 (2000) 9330.

[31] R.S. Knox, B.Q. Spring, Photochem. Photobiol. 77 (2003) 497. 